\title{
Interstitial brachytherapy in soft tissue sarcoma: a 5 years institutional experience with Cobalt 60- based high-dose-rate brachytherapy system
}

\author{
Kazi Sazzad Manir, MD, DNB!, Abhishek Basu, MD, DNB², Krishnangshu B. Choudhury, MD², Swapnendu Basu, MD, DNB!' \\ Koushik Ghosh, MSc, Dip RP2, Subir Gangopadhyay, MD² \\ 'Department of Radiation Oncology, Medica Cancer Hospital, Siliguri, WB, ${ }^{2}$ Department of Radiation Oncology, R. G. Kar Medical College \\ and Hospital, Kolkata, WB, India
}

\begin{abstract}
Purpose: Soft tissue sarcoma (STS) is rare but aggressive neoplasm. Interstitial brachytherapy (ISBT) alone or combined with external beam radiotherapy (EBRT) as post-operative treatment improves loco-regional (LRC) and distant control.

Material and methods: Out of twenty-nine non-metastatic STS (lower limb 64\%) patients (median age 37 yrs), treated with surgery and post-operative ISBT during February 2011 - December 2016, 27 patients with > 6 months follow-up were analyzed. Spindle cell sarcoma was the commonest $(24 \%)$ histology. Eleven patients $(44 \%)$ received EBRT (45-50 Gy), where ISBT was used as boost (16-20 Gy). Fourteen patients (56\%) received ISBT alone (4 Gy per fractio). Treatment was done with a 60 Cobalt $\left({ }^{60} \mathrm{Co}\right)$ source high-dose-rate system.

Results: With a median follow-up of 20 months (17-51 months), LRC rate was $85.7 \%$ (with EBRT 90.5\% and ISBT $83.2 \%$ alone). Median disease-free survival (DFS) was $39.7 \pm 3.9$ months (32-47.2 months). Median loco-regional failure-free survival (LRRFS) was $43.8 \pm 3.6$ months (36.8-50.9 months). Distant failure-free survival (DFFS) was 18 months (15.5-26.6 months). Overall survival was $42.4 \pm 3.4$ months (35.7-48.1 months). Tumor grade was a significant factor for DFFS. Total radiation dose (including EBRT) has significant influence on DFS and LRRFS. 14.8\% patients developed $\geq$ grade 2 late toxicity (skin atrophy, hypo-pigmentation, and telangiectasia).

Conclusions: Combination of surgery and ISBT with/out EBRT improves local and distant control with acceptable late toxicities. ${ }^{60} \mathrm{Co}$-based ISBT is safe and gives a good outcome.

J Contemp Brachytherapy 2018; 10, 5: 431-438 DOI: https://doi.org/10.5114/jcb.2018.78994
\end{abstract}

Key words: Cobalt 60, interstitial brachytherapy, soft tissue sarcoma.

\section{Purpose}

Soft tissue sarcomas (STS) are comparatively rare heterogeneous group of tumors with different distinct histopathological subtypes. It accounts for $21 \%$ of all pediatric non-hematological malignancies, and less than $1 \%$ of all adult solid neoplasm [1]. Due to rarity of the incidence and heterogeneous pathological varieties, STS have aggressive biological behavior and high potential for local recurrence after surgery. Adjuvant radiation therapy (RT), either preoperative or post-operative, improves local control (LC) rates after local resection/ limp sparing surgery, eliminating the need for amputation in limb sarcoma $[2,3,4,5]$. Use of RT as single modality treatment option gives a similar outcome in comparison to surgery without any major functional impairment [5].
Brachytherapy (BT) delivers high-dose of radiation precisely to the target tissue with high conformity sparingly the nearby normal tissues, thereby escalating the dose and eliminating the possibility of normal tissue toxicities. Role of adjuvant post-operative BT with or without EBRT showed increased LC rate $[6,7,8,9,10,11,12,13]$. These retrospective series included patients from different age groups and tumor grades. BT was used either as single modality or as boost to EBRT in either radical or post-operative setting. Most common mode of BT delivery was interstitial brachytherapy (ISBT).

Despite the fact that Cobalt $60\left({ }^{60} \mathrm{Co}\right)$ and Iridium 192 ( ${ }^{192}$ Ir) as high-dose-rate brachytherapy (HDR BT) source have different physical characteristics, they have identical dose distributions and clinical impact as supported by different dosimetric and clinical studies [14,15,16,17]. Longer half-life of ${ }^{60} \mathrm{Co}$ sources (5 years vs. 73 days)
Address for correspondence: Kazi Sazzad Manir, MD, DNB, Radiation Oncology, Medica Cancer Hospital, Rangapani, Siliguri, Dist: Darjeeling, Pin: 7334434, WB, India, phone: +919007933709, $\bowtie$ e-mail: kazi.dr@gmail.com
Received: 06.03 .2018 Accepted: 21.09 .2018 Published: 30.10 .2018 
makes it logistically more advantageous in low resource countries. However, higher energy emitting ${ }^{60} \mathrm{Co}$ requires more radiation protection measures. Evidence of ISBT using ${ }^{60} \mathrm{Co}$ HDR source is very little $[17,18]$. Clinical evidences on ${ }^{60} \mathrm{Co}$ HDR source-based BT are mostly on cervical cancer intracavitary applications.

Initially, we reported phase 2 feasibility analysis of ${ }^{60} \mathrm{Co}$ HDR-based ISBT in different sites [18]. In the current study, we analyzed our 5 years institutional experience of ${ }^{60} \mathrm{Co}$-based HDR ISBT in STS (radical BT or BT boost).

\section{Material and methods}

\section{Study design and study population}

In this retrospective institutional trial, twenty-nine non-metastatic pathologically proven STS patients, irrespective of site and location of tumor (treated between January 2011 to December 2016 with multimodality approach) were included. Patients $<6$ months follow-up and patients having unplanned surgery were excluded from the study.

\section{Staging evaluation}

Before the treatment, all patient underwent either magnetic resonance imaging (MRI) or contrast enhanced computer tomography (CECT) of the local and regional, CECT thorax, and histopathological examination ( \pm immunohistochemistry) from biopsy specimen before planned surgery.

\section{Surgery}

All surgeries were planned and discussed in multidisciplinary team. Basic surgical principles were to acquire a R0 resection [19]. Minimum $2 \mathrm{~cm}$ free margins were maintained. Dissections were done through uncontaminated normal tissue planes. Biopsy scars (tattooed) were also removed and drains were placed as close as possible to incision lines.

\section{Brachytherapy}

Any high-grade tumor ( $\geq$ grade 2 ) with size $\geq 5 \mathrm{~cm}$ lesion was included as a candidate for post-operative radiotherapy [20]. In most of the cases, we attempted intra-operative catheter placement, especially in deep seated tumors. For selective cases, post-operative ISBT was completed (superficial tumor, R1/R2 resection after primary surgery). In situations, where tumor bed was directly related to neurovascular bundles or bones (where periosteum is removed), ISBT was not attempted.

In majority of cases, radiation oncologist team attended the surgery with surgical oncologist team. Surgical clips were placed after removal of the tumor to localize the clinical target volume (CTV). In most of patients, we placed BT catheter intra-operatively. Hollow plastic flexible catheters were placed with help of flexible steel needles through skin over the tumor bed either along or perpendicular to the incision line depending on the location and size of the tumor bed. Aimed distance between catheters was $1.5 \mathrm{~cm}$. Parallelism was maintained between catheters. Entry and exit points of the catheters were kept $2 \mathrm{~cm}$ away from incision line. We used catheters with one end open. Buttons/balls were placed in both ends of the catheters. Adequate gap of $5 \mathrm{~mm}$ was kept between end buttons and skin to encompass post-operative tissue edema. Catheters were finally inspected after closure of the skin to ensure parallelism and rule out any kinking of the catheters. We did planning based on CECT, placing dummy ribbons through catheters (to aid catheter reconstruction) with slice thickness of $2.5 \mathrm{~mm}$ on $5^{\text {th }}$ post-operative day. Clinical target volume was contoured with help of surgical clips, surgical photographs, preoperative imaging, and pathological information. Organs at risk (OAR) were also contoured, mostly bone and skin (Figure 1). According to our institutional protocol, we contoured $2 \mathrm{~mm}$ of skin thickness from external contour over $5 \mathrm{~cm}$ of the CTV margin. Catheter reconstructions were done by library method. This method is time saving and reduces the probability of catheter misidentification, specially dealing with large number of catheters [21]. CTbased planning and optimizations were completed using HDR plus treatment planning system (version 2.6, Eckert \& Ziegler BEBIG GmbH, Berlin, Germany).

For post-operative cases, post-operative CECT was done to localize surgical clips and tumor bed. Brachytherapy catheters insertions were done under regional anesthesia. Catheter geometry was planned considering surgical note, position of surgical clips, and pathological findings. Planning scans were taken on day 2 after insertion.

Dose prescription was 3.5-4 Gy per fraction. We evaluated CTV $\mathrm{D}_{90}$ (defined as the minimum dose covering $90 \%$ of the CTV volumes), CTV $\mathrm{V}_{100 \%}, \mathrm{~V}_{300 \%}$ (defined as percentage volumes of CTV receiving $100 \%$ and 300\% of prescription doses, respectively), and respective OAR doses. For skin, we evaluated maximum point doses and D10 cc dose (defined as maximum dose received by minimum $10 \mathrm{cc}$ volumes of skin) and $\mathrm{V}_{100 \%}$ doses. We calculated EQD2 (defined as equivalent dose in 2 Gy/fraction). For BT boost cases, provisional EBRT doses were also summarized. For plan evaluation, our protocol was to keep CTV $\mathrm{D}_{90}$ does to $100 \%$. For OAR, we followed published cut off guidelines. For skin, as there is no such reported guideline, we tried to keep maximum dose as low as possible and $\mathrm{d} 10 \mathrm{cc}$ to $<2 / 3^{\text {rd }}$ of $\mathrm{D}_{90}$ doses.

Adjuvant radical BT (without EBRT) was given for small $(\leq \mathrm{T} 2)$, superficial tumors, with R0/R1 resection. In other cases, EBRT was added. For radical ISBT, 12-13 fractions (of $4 \mathrm{~Gy} /$ fraction, 2 fractions daily, 6 hours apart) were prescribed (EQD2 around $60 \mathrm{~Gy}$ ). For BT boost, 4-5 fractions (EQD2 around 16-20 Gy) were prescribed. Treatment delivery was given using MultiSource ${ }^{\circledR}$ (Eckert \& Ziegler BEBIG GmBH, Berlin, Germany), HDR remote after-loader system with ${ }^{60} \mathrm{Co}$ source.

\section{External beam radiation therapy}

In ISBT boost cases, EBRT was administered after 3 weeks of ISBT. For cases where intra-operative catheter placement was not done, we attempted earlier initiation of EBRT. Planning scan was done with $2.5 \mathrm{~mm}$ slice thickness. CTV was defined considering preoperative imag- 

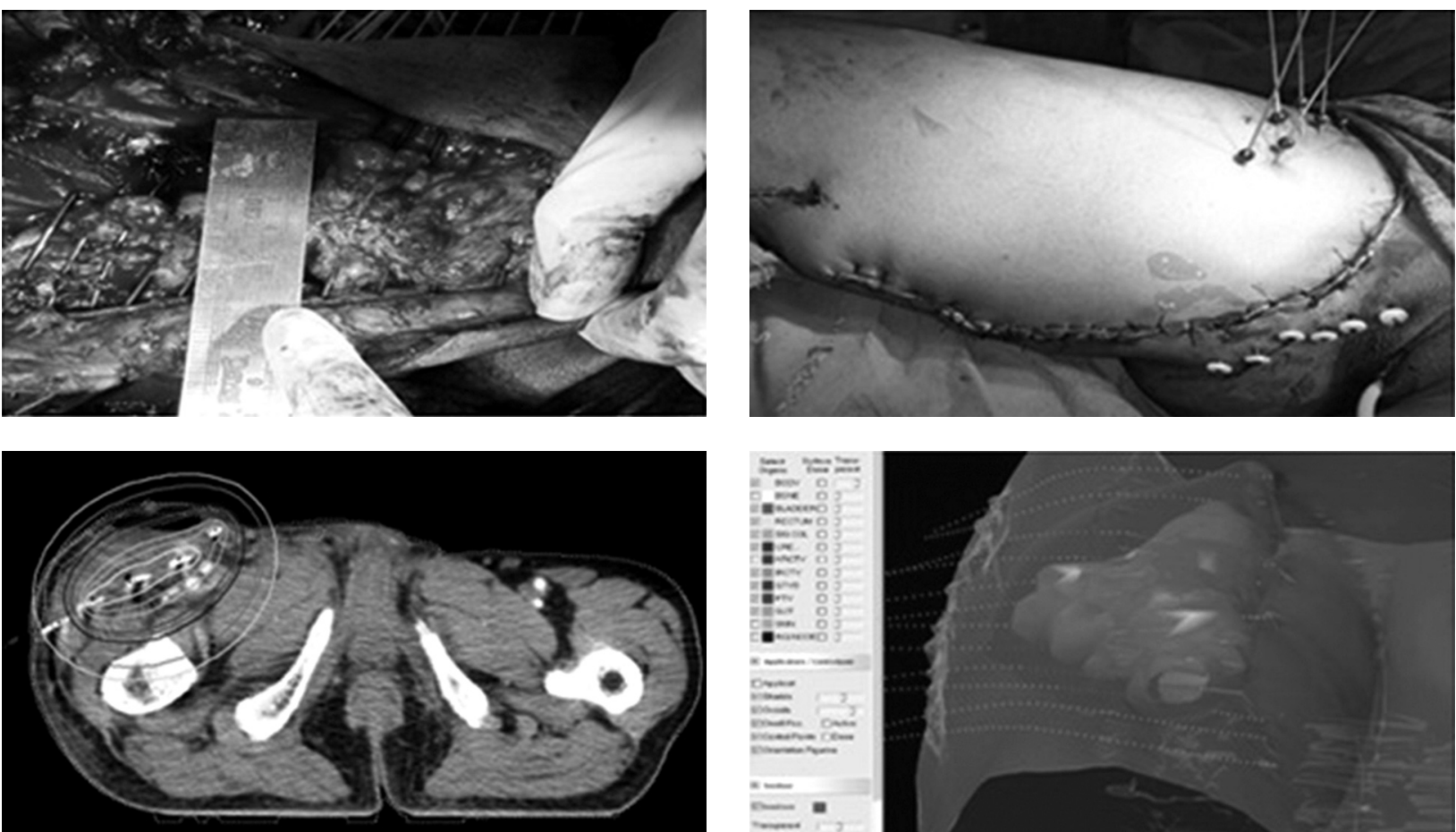

Fig. 1. ISBT process (steps are depicted sequentially from upper left, upper right lower left, and lower right figure). Upper left figure demonstrates per operative catheter placement in tumor bed maintaining parallelism equidistantly. Upper right figure shows the site after closure of wound with the BT catheters in situ. Lower right figure shows two dimensional isodose distribution. Lower left figure shows three dimensional isodose distribution with respect to reconstructed catheters and CTV

ing, ISBT CTV, and pathological findings. Approximately $5 \mathrm{~cm}$ CTV margins were applied. At least $1.5-2.0 \mathrm{~cm}$ of limb circumference was spared from radiotherapy portals. We tried to spare half circumference of uninvolved bone whenever possible and kept uninvolved compartment out of radiation port as far as possible. Threedimensional conformal RT (3D CRT) planning was done. Dose prescriptions were in the range of 45-50 Gy (1.8-2 Gy/fraction).

\section{Chemotherapy}

Anthracyclin and ifosfamide-based chemotherapy (ChT) was applied to chemo-sensitive histological subtypes. Total, 4-6 cycles of ChT was given. Injection filgrastim was administered routinely. Dose modification was done in patients with $\geq$ grade 2 hematological toxicities. In patients, where ChT was added as neo-adjuvant prior to surgery, patient was restaged by clinical and MRI findings. In adjuvant setting, ChT was given 2-3 weeks after completion of RT.

\section{Follow-up}

Patients were followed-up 2-3 monthly in initial 2 years and 6 months thereafter. During follow-up, patients were examined clinically. Chest $X$-rays were done in every follow-up. In suspicion of lung metastasis, CECT thorax was completed. Imaging of local sites were done depending on locations of the primary tumors. For R2 resection cases, in first follow-up visit, MRI or CECT was routinely done. MRI with or without contrast (or CECT) of the local site (every 6 months for 2 years, then annually) were completed for cases where loco-regional areas cannot be properly assessed by physical examination. In other cases, loco-regional imaging was done on clinical suspicion of recurrences. Toxicities, if occurred, were noted and managed accordingly. Progressive disease cases were managed as per institutional protocols. Late toxicities were measured using Common Terminology Criteria for Adverse Events (CTCAE) version 5.0 [22]. Grade 2 and higher-grade toxicities were considered as quantal end points for toxicity reporting.

\section{Statistical principle}

Statistical analysis was done using Statistical Package for the Social Sciences (SPSS) software, version 20 (IBM Corporation, USA). Descriptive statistics was done using frequency tables. Univariate analysis was completed to find out the impact of individual risk factors. Kaplan-Meier survival (KMS) plots were used to estimate overall survival (OS), disease-free survival (DFS), loco-regional failure-free survival (LRFFS), and distant failure-free survival (DFFS). Cox regression analysis was done to estimate of influence of individual factors on survival plots. $P$ value $<0.05$ was considered as statistically significant.

\section{Results}

Total twenty-nine eligible non-metastatic STS patients were treated between 2011-2016. Two patients were excluded from the analysis due to short follow-up ( $<6$ months), therefore final sample size was 27 patients. 


\section{Descriptive statistic}

Baseline characteristics of the patients are summarized in Table 1. Histopathologic subtypes are summarized in Table 2.

\section{Treatment}

Chemotherapy was given to $17(60.7 \%)$ patients. Nineteen $(70.4 \%)$ patients received radical ISBT, whereas

Table 1. Baseline characteristics

Baseline profile, $n$ (\%)

\begin{tabular}{lc}
\hline Median age & 38 years (10-73) \\
\hline Male & $15(53.6)$ \\
\hline Female & $12(42.9)$ \\
\hline Stage & $6(21.4)$ \\
\hline I & $17(60.7)$ \\
\hline III & $4(14.3)$ \\
\hline Grade & $4(14.3)$ \\
\hline 1 & $13(46.4)$ \\
\hline 2 & $10(35.7)$ \\
\hline 3 & $17(62.9)$ \\
\hline Site & $7(26)$ \\
\hline Trunk & $3(11.1)$ \\
\hline Upper limb & \\
\hline
\end{tabular}

$8(29.7 \%)$ patients received ISBT boost along with EBRT. Majorities $(17,62.9 \%)$ were post-operative ISBT implants. In ten cases $(37.1 \%)$, we did intra-operative catheter insertions. $19(70.4 \%)$ patients were treated by radical adjuvant ISBT (without EBRT) and in $8(29.6 \%)$ cases, ISBT boost was given along with adjuvant EBRT. In 15 (53.7\%), $2(7.2 \%)$, and $1(3.6 \%)$ cases we performed single, double, and triple plane catheter insertions, respectively.

\section{Dosimetric analysis}

Median EBRT dose was 50 Gy (30-50 Gy). Median ISBT prescription dose (per fraction) was 4 Gy (3-5 Gy). In radical ISBT (without EBRT), median fraction size was 12 (9-16) and in ISBT boost cases, it was 5 (3-6). Other dosimetric analyses are presented in Table 3 .

Table 2. Histological subtypes Histological types, $n(\%)(n=27)$

\begin{tabular}{lc}
\hline Chondrosarcoma & $2(7.2)$ \\
\hline Dermatofibrosarcoma protuberance (DFSP) & $3(10.7)$ \\
\hline Fibrosarcoma & $2(7.2)$ \\
\hline Leiomyosarcoma & $2(7.1)$ \\
\hline Malignant fibrous histiocytoma (MFH) & $1(3.6)$ \\
\hline Malignant peripheral nerve sheath tumor (MPNST) & $2(7.1)$ \\
\hline Myxofibrosarcoma & $1(3.6)$ \\
\hline Pleomorphic sarcoma & $4(14.3)$ \\
\hline Spindle cell ca & $6(21.4)$ \\
\hline Synovial cell ca & $2(7.2)$ \\
\hline STS NOS (no other specified) & $3(10.7)$
\end{tabular}

Table 3. Dosimetric analysis

\begin{tabular}{|c|c|c|c|c|c|}
\hline $\begin{array}{l}\text { ISBT/dosimetric } \\
\text { type }\end{array}$ & Parameters & Median (range) & Mean \pm SD & $\begin{array}{c}\mathrm{EQD}_{\alpha / \beta}=10 \\
\pm \mathrm{EBRT} \\
\text { Median (Gy) }\end{array}$ & $\begin{array}{c}\mathrm{EQD} 2_{\alpha / \beta}=10 \\
\pm \mathrm{EBRT} \\
\text { Mean } \pm \mathrm{SD}(\mathrm{Gy})\end{array}$ \\
\hline \multicolumn{6}{|l|}{ CTV dosimetry } \\
\hline \multirow[t]{3}{*}{ Radical ISBT } & $D_{90}$ & 4 Gy (3-5) & $4.1 \pm 0.5$ Gy & $60.7(33.4-67.4)$ & $55.3 \pm 9.4$ \\
\hline & $V_{100}$ & $84.8 \%(61.5-98)$ & $83.1 \pm 11.3 \%$ & - & - \\
\hline & $V_{300}$ & $7 \%(1.5-33.5)$ & $11.6 \pm 9.3 \%$ & & \\
\hline \multirow[t]{3}{*}{ Boost ISBT } & $\mathrm{D}_{90}$ & 4 Gy (3-5.5) & $3.3 \pm 0.7$ Gy & $66.6(42-78)$ & $66.1 \pm 11.4$ \\
\hline & $V_{100}$ & $91 \%(83.8-98.8)$ & $92.4 \pm 4.1 \%$ & - & - \\
\hline & $V_{300}$ & $7.6 \%(0.2-18)$ & $7.2 \pm 4.5 \%$ & - & - \\
\hline \multicolumn{6}{|l|}{ Skin dosimetry } \\
\hline & $\mathrm{D}_{10 \mathrm{cc}}$ & 2.8 Gy $(0.5-8.4)$ & $5.9 \pm 1.5$ Gy & - & - \\
\hline & $V_{100}$ & $17.8 \%(0-74.4)$ & $22.9 \pm 10.5 \%$ & - & - \\
\hline
\end{tabular}

ISBT - interstitial brachytherapy; $E Q D 2_{\alpha / \beta=10}$ - equivalent dose in $2 \mathrm{~Gy} /$ fraction; $S D$-standard deviation; $C T V$ - clinical target volume; $D_{90}$-defined as the minimum dose covering $90 \%$ of the CTV volumes; $V_{100 \%}, V_{300 \%}$ - defined as percentage volumes of CTV receiving $100 \%$ and $300 \%$ of prescription doses, respectively; $D_{10 c c}$ dose - defined as maximum dose received by minimum $10 \mathrm{cc}$ volumes of skin 


\section{Outcome (survival) analysis}

Survival analysis are summarized in Table 4. Figures 2-4 show KMS survival plots of LRFFS, DFS, and OS, respectively. With a median follow-up of 20 months (17-51 months), overall LC rates were $85.7 \%$ (BT boost: $90.5 \% /$ radical BT: $83.2 \%)$. LRRFS was $43.8 \pm 3.6$ months (36.8-50.9 months). DFS was $39.7 \pm 3.9$ months (32-47.2 months). Median DFFS and OS were $21.5 \pm 2.7$ months (15.9-26.6 months) and $42.4 \pm 3.4$ months (35.7-48.1 months), respectively.

On Cox regression analysis, tumor grade was found to be a significant factor on DFS and DFFS. Total EQD2 dose was significant factor for LRFFS and DFS. None of the dosimetric data had influence overall OS.

\section{Late toxicity analysis}

Late toxicities were reported using Common Terminology Criteria for Adverse Events (CTCAE) version 5.0. Twenty two $(81.4 \%)$ patients did not suffer any significant (grade 2 and higher) late skin toxicity. One (3.7\%) patient had grade 2 telangiectasia, $2(7.4 \%)$ patients had grade 2 skin hypo-pigmentation, and $1(3.7 \%)$ patient had grade 2 and grade 3 skin atrophy, respectively. No other late toxicity occurred during the follow-up period.

\section{Discussion}

This study is probably the first report of ISBT of STS using ${ }^{60} \mathrm{Co}$ HDR sources. We did this clinical audit to find out dosimetric and clinical outcome (in terms of local control, survival, and toxicities) of this HDR source.

HDR BT using ${ }^{60} \mathrm{Co}$ HDR sources has similar dosimetric and clinical outcome with commonly used ${ }^{192}$ Ir sources $[14,15,16,17]$. Clinical outcome data using ${ }^{60} \mathrm{Co}$ HDR sources in intracavitary BT has been published [17]. Our center is using ${ }^{60} \mathrm{Co}$ HDR-based BT systems since 2011.

Table 4. Outcome (survival) analysis

\begin{tabular}{lc} 
Median follow-up & 20 months (17-51) \\
\hline Progression & $1(3.7 \%)$ \\
\hline LR recurrence & $3(10.7 \%)$ \\
\hline Lung metastasis & $1(14.9 \%)$ \\
\hline Liver metastasis & (3.7\%) \\
\hline Over all RR (5 yr) & $\begin{array}{c}75 \%(\text { BT boost: } 83.3 \% / \\
\text { radical BT: } 76.2 \%)\end{array}$ \\
\hline Local control (5 yr) & $\begin{array}{c}85.7 \%(B T \text { boost: } 90.5 \% / \\
\text { radical BT: 83.2\%) }\end{array}$ \\
\hline Survival (months) & Mean \pm SE (95\% CI) \\
\hline LRFFS & $43.8 \pm 3.6(36.8-50.9)$ \\
\hline DFS & $39.7 \pm 3.9(32-47.2)$ \\
\hline Distant FFS & $21.5 \pm 2.7(15.9-26.6)$ \\
\hline OS & $42.4 \pm 3.4(35.7-48.1)$
\end{tabular}

$R R$ - response rate; SE - standard error of mean; LRFFS - loco-regional failure-free survival; DFS - disease-free survival; distant FFS - distant failure-free survival; OS - overall survival; yr-years

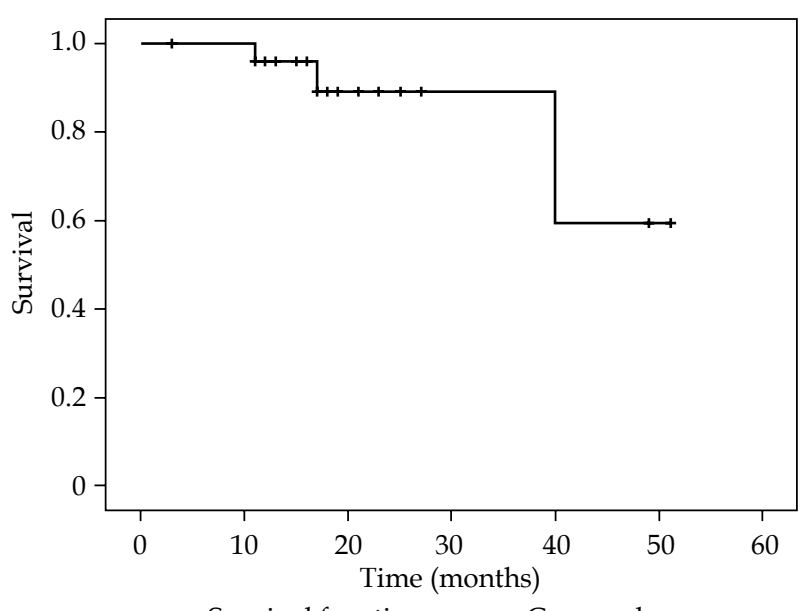

$\rightarrow$ Survival function $\quad+$ Censored

Fig. 2. Kaplan-Meier survival plot showing loco-regional failure-free survival

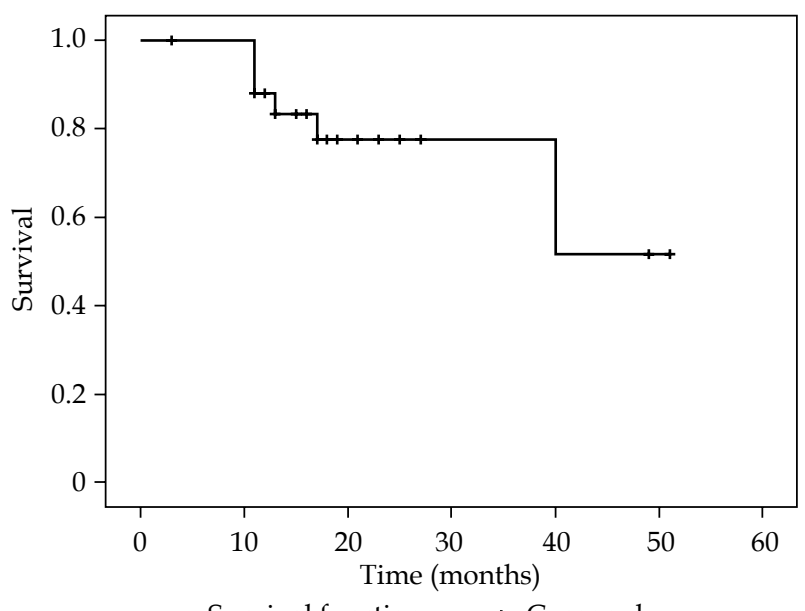

$\neg$ Survival function + Censored

Fig. 3. Kaplan-Meier survival plot showing disease-free survival

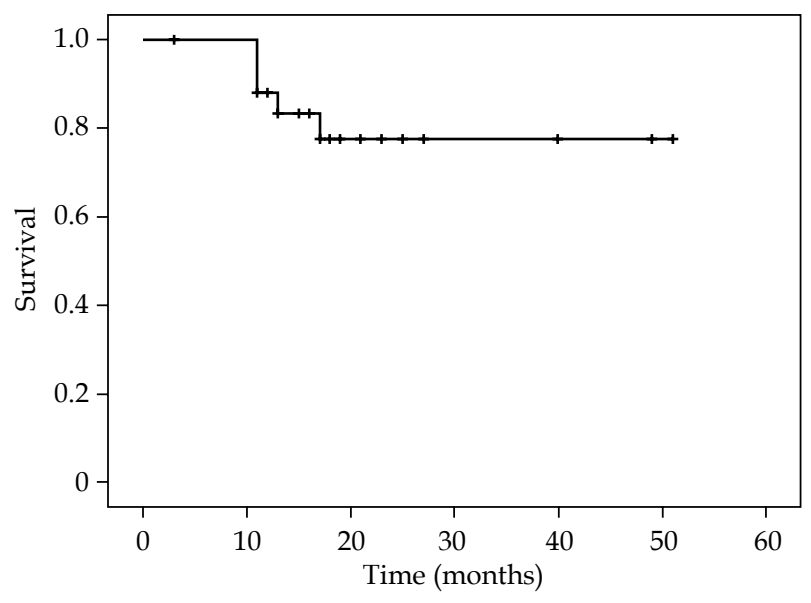

$\neg$ Survival function + Censored

Fig. 4. Kaplan-Meier survival plot showing overall survival 


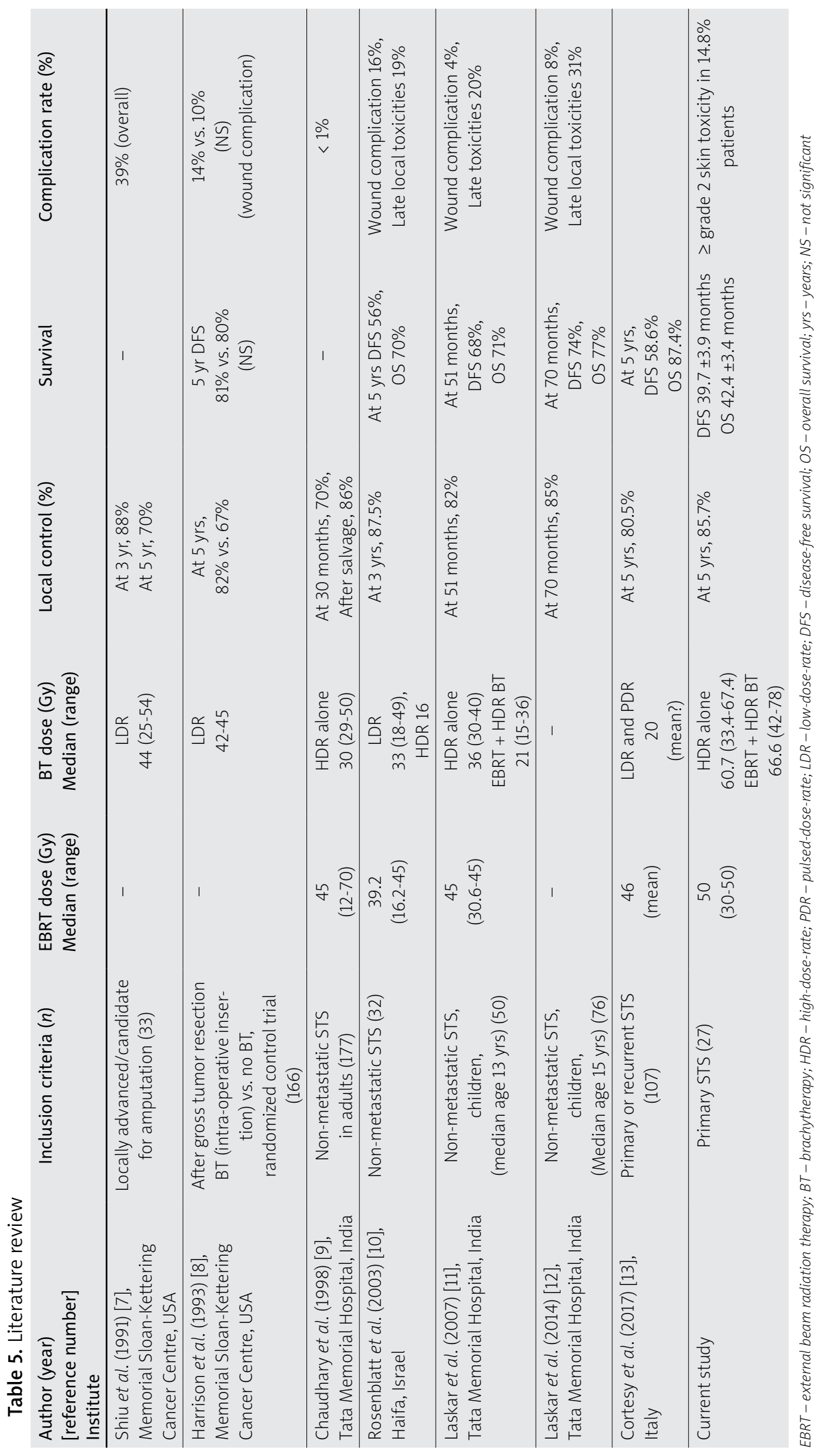


In 2012, we published initial experience of ISBT in different sites using this system [18]. It was phase I feasibility study and first of its kind. In this study, we summarized first three months follow-up (from October 2010 to December 2011) of 31 patients with carcinoma of different sites, treated at our center with ${ }^{60} \mathrm{Co}$ HDR BT with interstitial implants or surface molds with or without EBRT. We showed that HDR BT interstitial implants or surface molds using ${ }^{60} \mathrm{Co}$ sources for different sites is clinically feasible and safe with favorable short-term response and toxicity. It is also a very viable option for economically strained regions as for the half-life of ${ }^{60} \mathrm{Co}$.

Adjuvant BT is an important component of multimodality management of non-metastatic STS. ISBT using ${ }^{192}$ Ir sources $( \pm$ EBRT) as adjunct to radical surgery improves LC $[6,7,8,9,10,11,12,13]$. In STS, ISBT has many advantages over EBRT. ISBT catheters can be directly placed over respected tumor bed during operation. Therefore, high-dose can be precisely delivered to the area containing microscopic disease. Sharp and rapid dose fall off in BT helps relatively spare the nearby normal tissue. Successful results of ISBT depends on proper imaging, preferably R0 surgery, placement of surgical clips over margin, and proper technique of ISBT catheter insertion covering surgical bed with adequate margin.

Experience of ISBT was accumulated over the years worldwide, with excellent outcome results and acceptable toxicities. Use of ISBT as single modality RT technique for STS in children is well established [11,12]. Post-operative ISBT with small margin avoiding EBRT has a good local control rate, with proper sparing of growing bones and soft tissues. Outcome and toxicity results of some of recent published works of ISBT in STS are detailed in Table 5. In this table, we also compared outcome of our study. All the patients in our study $(n=27)$ are of primary STS. We found a comparable LC rate (after 5 years follow-up) with previous reported series.

Like other studies $[11,12,13]$, tumor grade influenced the LC, DFS, and DFFS with grade 1 tumors having better prognosis than grade 2 and 3. However, there was no difference in OS. Groups receiving ISBT alone versus groups receiving ISBT + EBRT had comparable LC rates $(90.5 \%$ vs. $83.2 \%$, respectively; $p$ value $=0.21)$ corroborating results published by Laskar et al. [11]. Similarly, total EQD2 dose influenced LRRFS and DFFS like all previous reports. In our study, any of the co-factors had an effect over OS. It may be due to heterogeneity in patient population (age, grade, site, use of adjuvant ChT).

In our series, most of the patients had mild erythema and pain over ISBT sites for 7-10 days after RT, which resolved subsequently. $18.5 \%$ of patients had late toxicities, of which majorities were skin related $(14.5 \%$ had $>$ grade 2$)$. This finding is comparable to the earlier reports of ${ }^{192} \mathrm{Ir}$ source-based ISBT $[10,11,12,13]$.

There are few limitations of this study. This study is retrospective assessment in nature with small sample size. There is lack of homogeneity of radical/boost ISBT approach, chemotherapy use, and age among patients in this study. Nevertheless, all the patients were treated with approximately uniform doses (radical ISBT/EBRT + ISBT combination group-wise). Moreover, all the patients were followed-up by same team of radiation oncologists of our BT unit.

\section{Conclusions}

Excellent local control and disease-free survival with acceptable toxicities have been observed in ISBT \pm EBRT in STS patients using ${ }^{60} \mathrm{Co}$-based HDR system. Survival and toxicity outcomes are comparable with published series of HDR ISBT using ${ }^{192} \mathrm{Ir}$ sources. Results were persistent irrespective of age groups, histopathology, and grades. Tumor grade and total dose in terms of EQD2 are the two major factors influencing outcome. ${ }^{60} \mathrm{Co}$-based HDR ISBT is feasible, safe, and can be a good option for resource constraint countries for its longer half-life than ${ }^{192}$ Ir.

\section{Acknowledgement}

We are grateful to all staffs of brachytherapy unit of the study hospital for their kind contribution. We also express our gratitude to all our patients whose cooperation made the study complete.

\section{Disclosure}

Authors report no conflict of interest.

Source of support: R. G. Kar Medical College and Hospital, Kolkata, India.

Presented (e-poster) at Annual Conference of Association of Radiation Oncologists of India 2016, November 2016, Bhubaneswar, India.

\section{References}

1. Burningham Z, Hashibe M, Spector L, Schiffman JD. The epidemiology of sarcoma. Clin Sarcoma Res 2012; 2: 14.

2. Lindberg RD, Martin RG, Romsdahl MM, Barkley HT Jr. Conservative surgery and postoperative radiotherapy in 300 adults with soft-tissue sarcomas. Cancer 1981; 47: 2391-2397.

3. Suit HD, Proppe KH, Mankin HJ, Wood WC. Preoperative radiation therapy for sarcoma of soft tissue. Cancer 1981; 47: 2269-2274.

4. Eilber FR, Mirra JJ, Grant TT et al. Is amputation necessary for sarcomas? A seven-year experience with limb salvage. Ann Surg 1980; 192: 431-438.

5. Rosenberg SA, Tepper J, Glatstein E et al. The treatment of soft-tissue sarcomas of the extremities: prospective randomized evaluations of (1) limb-sparing surgery plus radiation therapy compared with amputation and (2) the role of adjuvant chemotherapy. Ann Surg 1982; 196: 305-315.

6. Hilaris BS, Shiu MH, Nori D et al. Limb-sparing therapy for locally advanced soft-tissue sarcomas. Endocuriether/Hyperthermia Oncol 1985; 1: 17-24.

7. Shiu MH, Hilaris BS, Harrison LB, Brennan MF. Brachytherapy and function-saving resection of soft tissue sarcoma arising in the limb. Int J Radiat Oncol Biol Phys 1991; 21: 1485-1492.

8. Harrison LB, Franzese F, Gaynor JJ, Brennan MF. Longterm results of a prospective randomized trial of adjuvant brachytherapy in the management of completely resected soft tissue sarcomas of the extremity and superficial trunk. Int J Radiat Oncol Biol Phys 1993; 27: 259-265.

9. Choudhury AJ, Laskar S, Badhwar R. Interstitial brachytherapy in soft tissue sarcomas The Tata Memorial Hospital experience. Strahlenther Onkol 1998; 174: 522-528. 
10. Rosenblatt E, Meushar N, Bar-Deroma R et al. Interstitial brachytherapy in soft tissue sarcomas: The Rambam experience. Isr Med Assoc J 2003; 5: 547-551.

11. Laskar S, Bahl G, Muckaden MA et al. Interstitial brachytherapy for childhood soft tissue sarcoma. Pediatr Blood Cancer 2007; 49: 649-655.

12. Laskar S, Khanna N, Puri A et al. Interstitial brachytherapy for childhood soft tissue sarcomas: Long-term disease outcome and late effects. Int J Radiat Oncol Biol Phys 2014; 90: S113-114.

13. Cortesi A, Galuppi A, Frakulli R et al. Adjuvant radiotherapy with brachytherapy boost in soft tissue sarcoma. J Contemp Brachytherapy 2017; 9: 256-262.

14. Richter J, Baier K, Flentje M. Comparison of ${ }^{60} \mathrm{Co}$ and ${ }^{192} \mathrm{Ir}$ sources in high dose rate afterloading brachytherapy. Strahlenther Onkol 2008; 184: 187-192.

15. Strohmaier S, Zwierzchowski G. Comparison of ${ }^{60} \mathrm{Co}$ and ${ }^{192}$ Ir sources in HDR brachytherapy. J Contemp Brachytherapy 2011; 3: 199-208.

16. Zakaria GA, Schütte W, Azhari HA. Dosimetry of HDR afterloading machines with Ir-192 and Co-60 sources: comparisons of different international protocols. Z Med Phys 2010; 20: 215-224.

17. Ntekim Al, Adenipekun AA, Akinlade Bl et al. High-doserate brachytherapy in the treatment of uterine cervical cancer using cobalt-60 radionuclide source: Three years treatment outcome. West Afri J Radiol 2014; 21: 21-25.

18. Basu S, Basu A, Ghosh K, Dutta S. A phase I feasibility study for HDR interstitial Brachytherapy using 60Co for different sites. Radiother Oncol 2012; 103 (Supplement 2): S166.

19. Wittekind C, Compton C, Quirkey P et al. A uniform residual (R) tumor classification. Cancer 2009; 115: 3483-3488.

20. Yang JC, Chang AE, Baker AR et al. Randomised prospective study of benefit of adjuvant radiation therapy in the treatment of soft tissue sarcomas of the extremity. J Clin Oncol 1998; 16: 197-203.

21. Otal A, Richart J, Rodriguez S et al. A method to incorporate interstitial components into the TPS gynecologic rigid applicator library. J Contemp Brachytherapy 2017; 9: 59-65.

22. https://ctep.cancer.gov/protocolDevelopment/electronic_applications/ctc.htm\#ctc_50. Assessed on $5^{\text {th }}$ September 2018. 